\section{L'Actualité économique}

L'ACTUALITÉ ÉCONOMIQUE

\section{Le Canada et G.A.T.T.} Bilan d'une politique

\section{Jacques Parizeau}

Volume 37, numéro 2, juillet-septembre 1961

URI : https://id.erudit.org/iderudit/1001634ar

DOI : https://doi.org/10.7202/1001634ar

Aller au sommaire du numéro

Éditeur(s)

HEC Montréal

ISSN

0001-771X (imprimé)

1710-3991 (numérique)

Découvrir la revue

Citer cet article

Parizeau, J. (1961). Le Canada et G.A.T.T. Bilan d'une politique. L'Actualité économique, 37(2), 238-252. https://doi.org/10.7202/1001634ar
Ce document est protégé par la loi sur le droit d'auteur. L'utilisation des services d'Érudit (y compris la reproduction) est assujettie à sa politique d'utilisation que vous pouvez consulter en ligne.

https://apropos.erudit.org/fr/usagers/politique-dutilisation/
Cet article est diffusé et préservé par Érudit.

Érudit est un consortium interuniversitaire sans but lucratif composé de l’Université de Montréal, l'Université Laval et l'Université du Québec à Montréal. Il a pour mission la promotion et la valorisation de la recherche. https://www.erudit.org/fr/ 


\title{
Le Canada et G.A.T.T.
}

\author{
Bilan d'une politique
}

L'expérience du Canada avec G.A.T.T. fut jusqu'à un certain point le développement d'une politique qui avait pris naissance plusieurs années auparavant. En effet, au moment où le gouvernement libéral arriva au pouvoir en 1935, il était devenu évident que les politiques très protectionnistes des années antérieures avaient servi leur fin et qu'un renversement de tendance était inévitable. Le traité commercial entre le Canada et les Etats-Unis qui entra en vigueur en 1936, fut la première étape vers une libéralisation du commerce et une réduction des barrières tarifaires. $\bar{A}$ ce point de vue, l'accord de 1936 impliquait une réduction considérable dans la protection jusqu'alors accordée à l'économie canadienne.

En 1938, on élargit cet accord par un accroissement des avantages garantis par chacun des deux pays.

La guerre réduisit dans une bonne mesure la valeur de ces aiscussions tarifaires, puisque des mesures plus directes assurèrent le contrôle de l'économie. Aussi est-il intéressant de noter que ce fut durant la guerre que l'on fit la première tentative majeure de supprimer les droits sur les mêmes produits manufacturés de chaque côté de la frontière. En effet, comme on le sait, les gouvernements canadien et américain abolirent leurs droits respectifs sur la machinerie agricole.

$\bar{A}$ la fin de la guerre, les courants commerciaux traditionnels sont largement obstrués et des contrôles directs empêchent la plupart des mouvements de marchandises et de capitaux. Il n'est

1. Traduction de la communication de l'auteur à la réunion des Écoles d’administration tenue à Montréal, en juin 1961. 
point nécessaire de décrire longuement ici l'état des relations internationales à cette époque. Cependant, il est peut-être utile de rappeler quelques aspects de cette période en ce qui concerne principalement le Canada, à cause de leur influence sur les politiques d'après-guerre de son gouvernement.

Quand la guerre se termine, le Canada semble enfin avoir atteint une apparente maturité économique. Il est devenu exportateur de capitaux et est alors en position de vendre à l'étranger d'importantes quantités de produits manufacturés hautement diversifiés. Effectivement, il est plutôt étonnant de constater qu'à cette époque, le Canada exportait des locomotives au Pakistan ou des navires au Brésil.

Naturellement, la pénurie de denrées de toutes sortes qui prévalait alors dans le monde ne durerait pas éternellement et on pouvait prévoir qu'il ne serait pas facile de garder de tels marchés. Mais on pensait pouvoir maintenir une partie des positions nouvellement gagnées.

C'est ainsi, par exemple, que les conditions favorables consenties à la Grande-Bretagne à l'époque des négociations sur le contrat de blé, furent offertes dans l'espoir que l'Angleterre laisserait la porte ouverte à quelques importations de produits. manufacturés.

Naturellement, la majorité des exportations canadiennes se composait encore de produits primaires dont les marchés demeuraient toujours aléatoires en raison de la pénurie de dollars dans de nombreux pays et du développement de la capacité de production dans plusieurs régions du monde. Il était donc dans l'intérêt direct du Canada de poursuivre la politique de libéralisation des échanges qui avait débuté avant la guerre.

Parmi les programmes arrêtés pour libérer le commerce, G.A.T.T. (General Agreement on Tariffs and Trade) avait à l'origine une dimension limitée et une origine douteuse. Sousproduit de l'Organisation du Commerce International qui ne connut qu'une brève existence, sa première manifestation fut la conférence de Genève de 1947 qui entérina une série de réductions des droits de douane et promulgua un code de bonne conduite pour ses membres. Ce fut au départ une sorte d'arrangement très lâche consistant en des mesures précises et explicites dans le cas 
des tarifs douaniers, mais beaucoup moins précises dans le cas des quotas qui étaient alors les véritables moyens de contrôle des échanges.

C'est ce qui explique l'acceptation des plus importantes réductions dans les tarifs douaniers entre 1947 et la conférence de Torquay de 1951. Il était d'autant plus facile d'accepter de telles réductions, la fixation des droits, le gel des marges préférentielles que très peu de pays faisaient usage de telles armes et que les contrôles des changes et des quotas régissaient en grande partie le commerce. Il ne faudrait pas croire, pour autant, que les négociations de G.A.T.T. furent inutiles. La contribution qu'elles apportèrent à la libéralisation des échanges était dans la bonne direction. Il n'en reste pas moins que des organisations aussi importantes que le Plan Marshall, l'O.E.C.E., l'Union Européenne des Paiements, le Fond Monétaire International, la Banque Internationale de Reconstruction, ont eu, dans l'immédiat après-guerre, une influence supérieure à celle de G.A.T.T.

La chute temporaire dans le taux de croissance économique, en 1949, aurait pu éventuellement remettre en évidence le problème des tarifs. Mais la guerre de Corée créa encore une fois des conditions de rareté et partout on connut une nouvelle croissance accélérée.

D'une façon générale, entre 1946 et 1952 ou 1953, les droits et, en général, les mesures de protection ne furent pas et ne pouvaient devenir un conflit politique dans plusieurs pays. Les problèmes de balance de paiements furent la plupart du temps résolus par l'application de quotas, dont la valeur protectrice était secondaire. La reconstruction et l'arriéré de la demande de biens de consommation provoquèrent une expansion assez forte pour faire oublier la plupart, sinon tous les problèmes de protection.

On peut même affirmer que G.A.T.T. n'aurait probablement pas vu le jour si la possibilité d'une récession d'après-guerre et d'un retour aux guerres de tarifs n'avait pas impressionné une importante partie de l'opinion publique.

Cependant, une convertibilité de facto des devises européennes s'établit graduellement; des quotas disparurent et les contrôles des changes s'assouplirent; depuis quelques années le degré de concurrence internationale s'est accru, les prix ont acquis une impor- 
tance plus considérable que naguère, et en corollaire, les tarifs et leuirs méthodes de calcul et d'application sont redevenus une préoccupation majeure. De même, on a eu tendance à accorder de plus en plus d'importance à la valeur de protection des restrictions quantitatives au fur et à mesure que s'aplanissaient les difficultés d'équilibrer les balances de paiements. Nous étions revenus au cœur des problèmes et des discussions si familières aux générations passées, et effectivement G.A.T.T. devint rapidement une très importante organisation.

Parallèlement, les membres de G.A.T.T. devinrent plus hésitants à concéder d'autres réductions de droits. L'intensification de la concurrence n'est pas la seule explication de cette attitude. On doit aussi tenir compte du nombre croissant des membres qui, grâce à la clause de la nation la plus favorisée, devenaient tous bénéficiaires du moindre avantage offert par l'un d'eux.

Cependant, on ne pouvait pas reprendre facilement ce qu'on avait déjà concédé, et l'organisation de nouveaux blocs commerciaux poussait ceux qui en étaient ou s'en voulaient exclus à soutenir G.A.T.T. et les principes sur lesquels il s'appuie. C'est ainsi que, en dépit du Marché commun européen ou de l'Association de libre-échange qui, en fait, ont créé une atmosphère entièrement nouvelle, G.A.T.T. a survécu. Quelques pays (comme le Japon) ont trop misé sur son existence pour l'abandonner d'un cœur léger.

Cette évolution générale des esprits à l'égard de G.A.T.T., tout au long des années d'après-guerre, est aisément perceptible au Canada. Il n'en reste pas moins que le Canada a trouvé dans cet organisme certains intérêts qui lui sont propres et une politique qui n'est pas tout à fait celle des autres pays.

Aussi, compte tenu de l'arrière-plan que nous venons d'esquisser, nous pouvons maintenant essayer de tracer une sorte de bilan des relations de notre pays avec l'Accord Général sur les Tarifs et le Commerce.

Les premières années de G.A.T.T. furent dans leur ensemble plutôt favorables au Canada pour des raisons très spéciales. Les concessions échangées avec la plupart des pays du monde furent d'une importance limitée à cause des autres barrières au commerce. 
Cependant, en échange de plusieurs réductions de droits de la part dụ Canada; les États-Unis réduisirent, d'une façon appréciable, quelques uns des leurs, d'une importance spéciale pour notre pays. Et nos voisins n'étaient pas entourés de restrictions quantitatives comme le Canada et l'Europe. Aussi, tout avantage tarifaire obtenu d'eux avait des répercussions immédiates sur les exportations canadiennes.

Par exemple, on réduisit de moitié les droits américains sur l'aluminium, et il en fut ainsi pour les droits sur le plomb, le nickel, le zinc et le bois. Les produits de la pêche bénéficièrent d'importantes réductions. De telles concessions ont joué un rôle considérable à un moment très critique.

En effet, le réseau des courants commerciaux canadiens se réorganise durant les premières années d'après-guerre. $\bar{A}$ cause de ses difficultés de réserves, la Grande-Bretagne ne pouvait plus garder sa situation relative comme l'un des deux principaux marchés pour les exportations canadiennes. De plus, une fois passée la pénurie de vivres en Europe, la discrimination contre les denrées de la zone dollar fut la règle plutôt que l'exception. Aussi les Etats-Unis devinrent le seul marché capable d'absorber sans entrave les surplus de la production canadienne. Les réductions dans les tarifs américains sur les produits primaires tels que les minéraux, le bois et les produits de la pêche facilitèrent considérablement la réorganisation du commerce d'exportation du Canada.

Ces réductions aidèrent, en outre, le rétablissement de la situation des réserves de devises étrangères à un moment où le déficit des paiements à l'égard des États-Unis ne pouvait pas être compensé par des entrées suffisantes de devises convertibles venant des autres pays.

En conséquence, il faut considérer que durant la période de transition d'après-guerre, les négociations de G.A.T.T. furent d'une valeur inestimable pour le Canada. Pendant un certain temps, des restrictions quantitatives et un contrôle des changes annulèrent largement ce que l'on accordait, tandis que ce que l'on recevait servait à réorganiser une structure du commerce et des paiements qui était devenue inconfortable voire dangereuse.

Un tel accord sembla si utile qu'il n'est pas surprenant de constater que, jusqu'à la conférence de Torquay de 1951, les con- 
cessions acceptées par le Canada ne suscitèrent que très peu d'oppo sition de la part de l'opinion publique et même du milieu des affaires.

Mais les années ont lentement et graduellement changé une telle attitude. Parce que le processus fut graduel, parce que jusqu'à récemment, personne ne comprit ce qui semble maintenant être un problème majeur, il peut être utile d'examiner certaines des clauses du G.A.T.T. aussi bien que les attitudes du gouvernement canadien à leur sujet, afin de voir dans quel sens leur application a contribué aux difficultés de l'heure actuelle.

Selon le processus des négociations adopté par G.A.T.T., une concession accordée par un pays donné peut prendre l'une ou l'autre des deux formes suivantes: la fixation ou la consolidation d'un droit pendant un certain temps, ou la réduction du droit. De plus, l'Accord stipule qu'on ne peut, en aucun cas, augmenter les marges de préférences. En outre, selon le principe de la nation la plus favorisée, tous les adhérents bénéficient automatiquement de toute concession garantie à l'un des membres par un autre. En conséquence, le nombre et l'identité des participants influencent directement l'importance et la répercussion d'une concession. Enfin celle-ci peut être annulée en vertu de la clause échappatoire si les importations compromettent sérieusement l'industrie nationale.

Voilà en gros les règles du jeu quant aux tarifs. D'autre part, les quotas n'étaient pas au début matière à marchandage. On admit leur imposition afin d'équilibrer les balances de paiements. Mais leur disparition graduelle était une fin en elle-même et non une forme de concession.

Comment le Canada interpréta-t-il de telles règles? Pour élucider cette question, il faut se rappeler certaines caractéristiques essentielles du tarif canadien. Après la guerre, le tarif se composait aussi bien de droits ad valorem que de droits spécifiques, et quelquefois des deux. Certains droits variaient selon le prix de la denrée, ou bien une valeur spécifique limitait le montant du droit à payer. Aussi, il y avait nécessairement deux forces différentes qui abaissaient la valeur protectrice des droits: d'une part, les réductions concédées dans les tarifs eux-mêmes et d'autre part, la diminution de l'équivalent ad valorem des droits spécifiques due à l'inflation. 
Si le gouvernement canadien avait voulu réduire la baisse généralisée dans la protection, il aurait négocié les droits spécifques ou la partie spécifique du droit. Ainsi, il aurait concédé des avantages que l'inflation transférait de toute façon à l'étranger, quel que soit le taux. Mais c'est le contraire qui se produisit. Les réductions affectèrent principalement le groupe des tarifs ad valorem et nombre de droits spécifiques furent gelés. Cette politique ne relevait cependant pas seulement de la politique commerciale générale du gouvernement canadien. On doit admettre que les pays étrangers n'étaient pas particulièrement intéressés à recevoir des concessions sur des tarifs spécifiques à un moment où le mouvement des prix mondiaux était rapidement à la hausse.

$\bar{A}$ tout événement, la politique suivie par le gouvernement eut de sérieuses implications. Tandis que le négociateur pouvait accepter des réductions de tarifs ad valorem sur une base parfaitement rationnelle et en pleine connaissance de cause, la fixation des droits spécifiques impliquait que le gouvernement canadien perdait tout contrôle sur la valeur protectrice de tels droits. $\bar{A}$ ce point de vue, une telle concession était largement irrationnelle.

De nos jours, il est clair que nous aurions dû transformer ces droits spécifiques ou ces parties spécifiques du droit en tarifs ad valorem avant de les geler. En fait, au fur et à mesure que les années passaient, le gouvernement accepta une baisse de la moitié ou même plus que la moitié de la valeur protectrice de tels droits. Telle fut, en définitive, l'orientation de l'ensemble de la politique commerciale à cette époque. Pour ne mentionner qu'un secteur, on modifia complètement la protection de l'industrie textile qui était jadis défendue par de nombreux droits spécifiques ou des limites spécifiques. Ainsi, cette industrie canadienne dut fonctionner non seulement contre un niveau de protection beaucoup plus bas, mais contre une structure de droits largement irrationnelle et déformée.

Si la réduction des droits ad valorem est l'aspect le plus évident de la participation du Canada au G.A.T.T., il n'est pas facile de se représenter l'étendue de telles réductions. Des concessions peuvent être spectaculaires mais avoir peu d'importance quant au commerce réel. Il est d'usage courant de réduire des droits tombés en désuétude ou s'appliquant à des produits insignifiants. D'autre part, on peut considérer l'importance des tarifs à deux 
points de vue: soit parce qu'ils s'appliquent à des montants considérables d'importations, soit parce qu'au contraire, ils empêchent des mouvements appréciables de denrées.

Ainsi, on ne peut pas juger, d'une manière entièrement rationnelle, les droits caractéristiques d'une structure tarifaire. Il faut choisir les taux dont l'expérience révèle qu'ils peuvent avoir plus d'importance que d'autres.

Nous avons fait un tel choix parmi 192 droits tirés de la liste des droits de la nation la plus favorisée et du tarif préférentiel. Il est nécessaire de considérer les deux listes dans la mesure où une réduction du tarif de la N.P.F. peut, dans plusieurs cas, produire une réduction correspondante dans le tarif préférentiel afin de maintenir la marge au même niveau. Nous avons choisi 1939 plutôt que 1946 comme année de base, plusieurs droits ayant été temporairement suspendus ou modifiés au cours des deux années qui suivirent la fin des hostilités. L'échantillon inclut donc l'abolition des droits sur l'équipement agricole qui, bien entendu, ne fut point négociée au sein du G.A.T.T. Puis, nous avons comparé ces droits d'avant-guerre à ceux de 1956, puisque nous savons, d'autre part, que le gouvernement modifia sa politique commerciale dans les années qui suivirent.

Voici les résultats de cette comparaison: on constate une réduction de 42 p.c. de tous les droits tandis que 58 p.c. demeurent inchangés (tableau 1). Parmi les droits réduits, 60 p.c. sont abaissés de moins de 20 p.c. de leur niveau original.

Si on analyse seulement la liste de la nation la plus favorisée, on peut estimer à 62 p.c. le nombre de postes réduits, tandis que 65 p.c. de ceux-ci sont diminués de 20 p.c. et moins.

Ainsi, il semble clair qu'on a abaissé les marges de préférences et que les réductions furent loin d'être aussi généralisées ou aussi importantes qu'on le croit généralement. Néanmoins, elles ont joué un rôle important dans la diminution générale de la protection depuis la guerre.

Cependant, une étude directe des tarifs ne donne pas une idée exacte de la mesure des concessions accordées par le Canada. Car plusieurs pays adhèrent maintenant au G.A.T.T., avec lesquels le Canada n'avait pas signé de traité commercial depuis plusieurs années. Cela a considérablement élargi l'application des concessions. 


\section{L'ACTUALITÉ ECONOMIQUE}

Tableau I

Diminution de certains droits de douanes entre 1939 et 1956

\begin{tabular}{|c|c|c|c|c|c|c|c|c|c|c|c|c|c|c|}
\hline \multicolumn{2}{|c|}{$\begin{array}{l}\text { Nombre de } \\
\text { diminutions }\end{array}$} & \multirow[t]{3}{*}{$\begin{array}{c}\text { Diminutions } \\
\text { en p.c. des } \\
\text { droits de } 1939\end{array}$} & \multicolumn{8}{|c|}{ Diminutions absolues des droits ${ }^{1}$} & \multicolumn{2}{|c|}{$\begin{array}{c}\text { Augmen } \\
\text { tations }\end{array}$} & \multicolumn{2}{|c|}{ Autres } \\
\hline \multirow{2}{*}{$\begin{array}{c}\text { Tarif } \\
\text { préfé- } \\
\text { rentiel } \\
\text { (P) }\end{array}$} & \multirow{2}{*}{ N.P.F. } & & \multicolumn{2}{|r|}{$21 / 2$} & \multicolumn{2}{|r|}{5} & \multicolumn{2}{|c|}{$71 / 2$} & \multicolumn{2}{|c|}{$\begin{array}{l}10 \text { et } \\
\text { plus }\end{array}$} & \multirow{2}{*}{$\mathrm{P}$} & \multirow{2}{*}{ N.P.F. } & \multirow{2}{*}{ P } & \multirow{2}{*}{ N.P.F } \\
\hline & & & $\mathrm{P}$ & N.P.F. & $P$ & N.P.F. & $\mathrm{P}$ & N.P.F. & $\mathrm{P}$ & N.P.F. & & & & \\
\hline \multirow[t]{7}{*}{74} & 35 & 5 à 10 p.c. & 1 & 17 & . & & & & & & 1 & 1 & 3 & 4 \\
\hline & & 11 à 15 p.c. & 6 & 3 & & 2 & & & & & & & & \\
\hline & & 16 à 20 p.c. & 1 & 1 & 3 & 14 & & & & 1 & & & & \\
\hline & & 21 à 25 p.c. & & 1 & 2 & 1 & & 2 & & & & & & \\
\hline & & 26 à 30 p.c. & & & & & & 2 & & 2 & & & & \\
\hline & & 31 p.c. et plus & & & & & & 2 & 1 & 5 & & & & \\
\hline & & non classifiés & 1 & 4 & 1 & 1 & & $1^{\circ}$ & & $\cdot$ & & & & \\
\hline 74 & 35 & Total & 9 & 26 & 6 & 18 & 0 & 7 & 1 & 8 & 1 & 1 & 3 & 4 \\
\hline
\end{tabular}

Parmi les cas de ce genre, le plus frappant est celui du Japon. Ici encore, la formule adoptée illustre bien jusqu'où notre gouvernement était prêt à aller dans sa politique de libéralisation des échanges. Bien que plusieurs membres de G.A.T.T. n'aient pas accepté le Japon comme un membre à part entière de l'organisation et continuent d'exercer une discrimination partielle contre ses produits, le Canada accorda tous les avantages de la clause de la nation la plus favorisée au nouveau membre du club; et cela, en dépit du fait que notre pays admettait ainsi les produits japonais à des taux souvent réduits de plus de la moitié.

Là encore, on avait pour principe que la possibilité de nouvelles exportations vers le Japon et plus particulièrement des exportations de blé, feraient plus que compenser les avantages qu'on lui accordait.

Quant aux quotas, la fin des problèmes de balance de paiements éprouvés par le Canada marqua aussi la fin des restrictions quantitatives pendant plusieurs années. Le gouvernement de cette époque se refusait à employer des quotas pour protéger un secteur donné de l'activité économique.

1. Représentant la différence entre le taux de 1939 et celui de 1956. 
Cette attitude si libérale fut aussi caractéristique de l'administration des douanes. On sait que le taux d'un droit peut avoir une valeur protectrice plus ou moins grande selon la base de sa compilation. Avant la guerre, il était d'usage courant au Canada d'utiliser la valeur de la facture comme base de compilation du droit, seulement si elle n'était pas plus basse que le coût de production. On convertissait alors la valeur de la facture en dollars canadiens au taux de change du marché, seulement si on jugeait que la devise étrangère n'avait pas été dépréciée pour donner un avantage non mérité à l'exportateur. Autrement, le Ministre pouvait établir un taux de change arbitraire afin de convertir la valeur de la facture.

G.A.T.T. a mis au point des règles très strictes à suivre pour ce qui est de l'évaluation. Les douaniers doivent utiliser la juste valeur marchande. Ce principe, extrait de son jargon, signifie qu'aux douanes, on doit toujours utiliser la valeur de la facture si, dans le pays d'origine, on vend des quantités équivalentes au même prix. Ceci semblait assez logique et les autorités canadiennes l'acceptèrent immédiatement. En procédant ainsi, on ouvrait naturellement une porte à des abus frappants qui provenaient du fait que les importateurs canadiens achetaient souvent des fins de course ou des lots de jobbers aux États-Unis à des prix très bas, dans plusieurs cas bien en dessous du coût de production de telles marchandises. Ceci est particulièrement vrai dans le cas de produits de qualité ou dont la présentation change souvent à cause de la mode ou d'une désuétude prévue. Et c'est ainsi qu'à la fin de la saison, les chutes de prix se multiplient, surtout à New-York et Chicago. Les importations taxées sur la base de la juste valeur marchande peuvent alors inonder le marché canadien au moment où la production canadienne de denrées similaires, habituellement légèrement en retard sur la fabrication américaine, est en pleine expansion.

Pour résumer, il est alors assez évident que les concessions garanties par le Canada furent importantes, quoique pas toujours très logiques, et ont contribué à une réduction appréciable de la protection. Comme on l'a souligné précédemment, cet état de choses ne rencontra pas d'opposition sérieuse jusqu'après la guerre de Corée. Cependant, depuis 1952, les difficultés croissantes de l'industrie secondaire canadienne et particulièrement 
d'industries spécifiques qui sont menacées de disparaître par l'accroissement massif des importations, ont suscité des demandes protectionnistes d'une intensité grandissante.

Sans doute, avec un retour graduel aux conditions normales de concurrence, la même chose arriva dans d'autres pays si bien que, comme on l'a déjà affirmé, les négociations de G.A.T.T. entrầnèrent de moins en moins de concessions de ses membres. Il est néanmoins remarquable que, pendant plusieurs années, le gouvernement canadien résista avec un grand succès aux pressions de toute part en vue d'ajuster ou d'accroitre la protection, même dans le cas d'industries individuelles. Il résista à un degré étonnant aux invitations d'invoquer la clause échappatoire de G.A.T.T. ou même d'élever des droits que n'avaient pas fixés des négociations antérieures. $\bar{A}$ quelques reprises, on s'efforça de faire accepter à certains pays des quotas volontaires, mais dans l'ensemble, la seule concession qui prit une certaine signification aux yeux de l'opinion publique fut d'utiliser éventuellement comme base de la juste valeur marchande la moyenne des prix durant les six derniers mois précédant les importations. De cette façon, on évitait d'utiliser les prix des jobbers ou de fins de course pour compiler les droits. La formule n'était pas entièrement satisfaisante, mais au moins contribuait-elle à corriger quelques abus notoires qui étaient apparus.

Cependant, quant au reste, et durant toutes ces années, le Canada fut le membre modèle de G.A.T.T., le grand champion de la libéralisation du commerce. Sans doute, le gouvernement canadien de cette époque savait bien que dans un monde où la concurrence grandit, un retour à la protection pourrait facilement bouleverser les marchés d'exportation pour les produits primaires canadiens. En conséquence, il mancuvra de telle sorte que le Canada n'ait pas la responsabilité de provoquer un tel retour à la protection. Á cet effet, on sacrifia des secteurs entiers de l'industrie manufacturière aux intérêts des industries exportatrices.

Tous les pays n'avaient cependant pas les mêmes intérêts ou les mêmes vertus. Les États-Unis, par exemple, conservèrent encore une protection massive contre les produits agricoles d'ori- 
gines étrangères. Ils utilisèrent la clause échappatoire à plusieurs occasions, soit pour retirer quelques concessions ou simplement pour désorganiser le commerce.

Selon G.A.T.T., on devait éliminer toutes les restrictions quantitatives sur les produits manufacturés avant 1957. En 1955 et 1956, il était loin d'être évident que l'objectif pouvait être atteint. Au contraire, les quotas étaient alors devenus l'objet de négociations et le Canada n'en avait aucun à négocier. Aussi devint-il graduellement évident que la place du Canada se faisait de moins en moins confortable à la table des négociations et qu'une attitude longtemps utile devenait gênante pour ne pas dire ridicule.

C'est alors que s'évanouirent nos derniers espoirs de conserver au moins l'essentiel de la politique suivie jusque-là.

La politique commerciale des Etats-Unis devint, en effet, de plus en plus dangereuse pour le commerce canadien d'exportation. La loi publique 480 qui favorisait considérablement les exportations américaines de blé dans les pays sous-développés porta un dur coup à nos propres ventes dans plusieurs régions du monde. Puis, après avoir investi des milliards de l'autre côté de leur frontière, les Etats-Unis restreignirent leurs importations de pétrole brut. Ils imposèrent par la suite des quotas sur les importations de zinc et de plomb, annulant ainsi les concessions qu'ils avaient accordées au début de G.A.T.T. Et encore plus récemment, les contrats d'uranium connurent une fin dramatique.

D'une manière moins spectaculaire, on ne pouvait plus considérer les marchés traditionnels pour les produits canadiens aux Etats-Unis comme appelés à prendre une aussi forte expansion dans l'avenir. Tel est le cas en particulier du marché pour le papier journal. En effet, tandis qu'au cours des sept ou huit dernières années la capacité de production a doublé aux Etats-Unis, les exportations canadiennes vers ce pays ont eu tendance à plafonner.

Heureusement, du côté de l'Europe, le rétablissement graduel de la convertibilité laissait espérer un développement des exportations canadiennes. Mais l'organisation du Marché commun: et l'Association de libre échange se constituèrent au moment même où le Canada pouvait enfin espérer profiter pleinement des avantages tarifaires qu'il avait accordés. La création de tels blocs va directement à l'encontre des principes de G.A.T.T. dans la mesure où. 
ils interdisent l'établissement de nouvelles préférences. Il était important pour les pays européens d'instituer ces nouvelles préférences et ils n'eurent pas de difficultés à faire accepter le principe par les membres de G.A.T.T. Cependant, le Canada, qui avait scrupuleusement observé les règles à cet égard et qui n'avait pas négocié de nouvelles préférences, même si à certains moments il aurait eu avantage à le faire, se retrouvait complètement à découvert.

Naturellement, dans l'ensemble, tous ces événements furent le signe de changements radicaux dans les marchés d'exportation du monde. Il était au moins certain que le Canada se devait de modifier la politique suivie jusqu'à ce jour. Car le fait de maintenir des positions fermes à l'égard des demandes des intérêts protectionnistes locaux ne provoquerait plus nécessairement ce qu'on avait toujours considéré comme un avantage marqué. Dix ans auparavant, l'Accord avait reçu un appui sérieux de ses membres, puis leur ardeur s'était refroidie; et maintenant plusieurs rejetaient sinon la lettre, du moins l'esprit des règles. La politique commerciale du Canada était mûre pour un changement et le changement du gouvernemént favorisa considérablement la transition.

Tout le monde sait maintenant que l'attitude qui fut adoptée pendant si longtemps n'existe plus. La politique tarifaire de notre pays a de nouveau fait preuve d'une grande flexibilité. Après l'arrivée au pouvoir des conservateurs, plusieurs droits négociés furent relevés contre la réduction d'autres, afin de pallier les diff. cultés d'industries particulières. Dans d'autres cas, si les droits demeurèrent les mêmes, on en changea la base d'évaluation. On rétablit le coût de production comme un minimum au-dessous duquel la juste valeur marchande ne pouvait plus descendre.

Au même moment, on entreprit un programme de simplification et de rationalisation du tarif afin de tenir compte des changements - dans les courants commerciaux et dans la technologie.

Enfin, récemment, la redéfinition de postes du tarif servit à remanier la protection de l'industrie de la machinerie, une initiative que le Sénat a d'ailleurs bloquée il y a quelques mois.

Des quotas ou des menaces de quotas ont fait également leur apparition pour la première fois depuis dix ans.

Toutes ces mesures soulignent deux caractéristiques de la politique commerciale actuelle. 
Premièrement, il est clair que le gouvernement a rétabli l'autonomie de son tarif. Une bonne partie du travail accompli durant les quelques dernières années s’apparente aux mesures prises par un bon nombre d'autres pays. En effet, les réformes s'accordent très mal sinon d'aucune façon avec les règles de G.A.T.T. Naturellement, le gouvernement canadien négocie avec les membres de telle sorte que ceux-ci acceptent les changements les plus désagréables sans trop de représailles.

En second lieu, la politique actuelle se dirige sans équivoque dans le sens d'un accroissement de la protection. On a, sans doute, manœuvré si habilement le changement qu'on l'a rarement pris pour ce qu'il était. Néanmoins, l'orientation fondamentale est assez évidente.

Certes, on connait bien la raison essentielle en faveur d'une protection accrue. On reconnaît de plus en plus que l'on a sacrifié les industries secondaires au bénéfice des industries exportatrices $\grave{a}$ un point tel qu'on peut douter que les avantages aient encore une valeur égale au coût.

Mais il peut bien y avoir une autre raison en faveur de la politique actuelle. Dans la mesure où se déroule un difficile marchandage entre les Etats-Unis et les blocs commerciaux qui sont en train de s'organiser en Europe, en Amérique latine et peut-être demain en Afrique, le Canada sera alors prêt à négocier en partant d'une position de force qui signifie à peu près toujours, dans les discussions de tarifs, une position de protection appréciable. On ne peut pas échanger ce qu'on n'a pas et un pays qui s'est aligné sur une grande liberté de commerce a peu de choses à offrir.

Il est inutile ici de chercher à savoir si la politique actuelle est justifiable ou si, parmi les nombreuses alternatives qui aujourd'hui ș'offrent au Canada, c'est la voie que nous devrions choisir. Il suffit de remarquer qu'un changement s'imposait et qu'effective. ment il s'est produit.

On peut d'ailleurs facilement constater qu'on a transformé la signification de G.A.T.T. au cours des dernières années aussi bien pour le Canada que pour plusieurs autres pays du monde. Désormais, ce n'est plus une organisation entièrement consacrée aux idéaux de la libéralisation des échanges. $\bar{A}$ cause des circonstances, G.A.T.T. est devenu de plus en plus un marché de droits et de 
quotas. Et comme dans le cas de n'importe quel marché, les droits peuvent être modifiés autant à la hausse qu'à la baisse. Cela implique-til pour autant que G.A.T.T. soit devenu une organisation de façade? Aucunement. Il peut encore jouer un rôle important comme siège de négociations ordonnées et comme une chambre de compensation de conflits et d'oppositions. On ne peut pas considérer cette fonction comme étant d'une importance mineure comme l'ont démontré d'autres organisations internationales. Si, cependant, des membres de G.A.T.T. insistaient pour que les principes originaux soient appliqués d'une façon stricte, alors il ne serait pas étonnant d'assister à la dislocation de cet organisme.

Cependant, quelle que grande que puisse être la flexibilité apportée dans les négociations, cela ne résoudra pas tous les problèmes. Dans les années à venir, les membres tendront sans doute à limiter plus étroitement la participation de plusieurs pays. Comme les régions sous-développées à bas salaire s'engagent dans un processus d'industrialisation basé sur la technologie la plus moderne, les membres actuels de G.A.T.T. devront s'efforcer de trouver une nouvelle sorte de relations commerciales avec eux. Il est très douteux que même un G.A.T.T. flexible puisse faire accepter les mêmes règles du jeu à des pays si différents quant à leurs conditions de production. Le Canada, aussi bien que plusieurs autres membres, éprouvera sans doute des difficultés à étendre sans restrictions le traitement de la nation la plus favorisée aux nouveaux venus. Et la dérogation à une telle clause automatique frapperait G.A.T.T. dans son essence même.

C'est pourquoi il est évident que si G.A.T.T. a été la pierre angulaire de la politique commerciale du Canada depuis la guerre, nous avons maintenant atteint le point où il nous faut trouver un système entièrement nouveau de rapports commerciaux et de techniques de négociations. Dans.ce sens, toute une époque vient de prendre fin.

Jacques PARIZEAU, professeur à l'Ecole des Hautes Etudes commerciales (Montréal). 